\title{
Distributed optimization on unbalanced graphs via continuous-time methods
}

DOI:

10.1007/s11432-018-9502-1

\section{Document Version}

Accepted author manuscript

Link to publication record in Manchester Research Explorer

\section{Citation for published version (APA):}

Li, Z., \& Ding, Z. (2018). Distributed optimization on unbalanced graphs via continuous-time methods. Science China. Information Sciences, 61(12), 1-3. [129204]. https://doi.org/10.1007/s11432-018-9502-1

\section{Published in:}

Science China. Information Sciences

\section{Citing this paper}

Please note that where the full-text provided on Manchester Research Explorer is the Author Accepted Manuscript or Proof version this may differ from the final Published version. If citing, it is advised that you check and use the publisher's definitive version.

\section{General rights}

Copyright and moral rights for the publications made accessible in the Research Explorer are retained by the authors and/or other copyright owners and it is a condition of accessing publications that users recognise and abide by the legal requirements associated with these rights.

\section{Takedown policy}

If you believe that this document breaches copyright please refer to the University of Manchester's Takedown Procedures [http://man.ac.uk/04Y6Bo] or contact uml.scholarlycommunications@manchester.ac.uk providing relevant details, so we can investigate your claim.

\section{OPEN ACCESS}




\section{VIVIIENCE CHINA \\ Information Sciences}

\section{Distributed Optimization on Unbalanced Graphs via Continuous-Time Methods}

Li Zhenhong and Ding ZhengTao

Citation: SCIENCE CHINA Information Sciences ; doi: 10.1007/s11432-018-9502-1

View online: http://engine.scichina.com/doi/10.1007/s11432-018-9502-1

Published by the Science China Press

\section{Articles you may be interested in}

A new look at the Lagrange method for continuous-time stochastic optimization SCIENCE CHINA Mathematics 55, 2247 (2012);

Difference graphs of a class of alternating block Crank- Nicolson methods

Chinese Science Bulletin 44, 1763 (1999);

Gibbs sampling based distributed OFDMA resource allocation

SCIENCE CHINA Information Sciences 57, 42302 (2014);

DIFFERENTIAL METHODS OF OPTIMIZATION

Chinese Science Bulletin 28, 281 (1983);

IDENTIFICATION OF LINEAR SYSTEMS WITHOUT ASSUMING STABILITY AND MINIMUM PHASE

Science in China Series A-Mathematics, Physics, Astronomy \& Technological Science 33, 641 (1990); 


\title{
Distributed optimization on unbalanced graphs via continuous-time methods
}

\author{
LI Zhenhong ${ }^{1} \&$ DING Zhengtao ${ }^{1 *}$ \\ ${ }^{1}$ School of Electrical and Electronic Engineering, University of Manchester, Manchester M13 9PL, UK
}

Citation Li Z and Ding Z. Distributed optimization on unbalanced graphs via continuous-time methods. Sci China Inf Sci, for review

Dear editor,

Distributed optimization problems (DOPs) have attracted significant attention in the past decade, due to their potential applications in a variety of scenarios such as sensor networks, distributed parameters estimation, and power system economic dispatch. An important class of DOPs refers to minimizing the sum of local objective functions (see, e.g., [1-3]):

$$
\min _{\omega \in \mathbb{R}^{n}} f(\omega)=\min _{\omega \in \mathbb{R}^{n}} \sum_{i=1}^{N} f_{i}(\omega),
$$

where $N$ is the number of agents, $f_{i}: \mathbb{R}^{n} \rightarrow \mathbb{R}$ is the local cost function of agent $i$, and $f=$ $\sum_{i=1}^{N} f_{i}(\omega)$ is the global cost function of the network.

To solve problem (1), two continuous-time schemes are designed from a control perspective in [4] to find the optimal solution with centralized and distributed structures, respectively. The distributed scheme achieves asymptotic convergence for constrained optimization problem on directed graphs. For the system with twice differentiable local cost functions, zero-gradient-sum method in [5] achieves exponential convergence if the initial value of states are the optimal solution of local cost functions. To remove the restriction on the initial condition, Lagrangian based algorithms are proposed in [2]. A remarkable feature of Lagrangian based algorithms is the use of auxiliary states which can also be regarded as Lagrangian multipli- ers. However the algorithms in [2] need to transmit the auxiliary states over the network. To reduce the communication cost, a new Lagrangian based algorithm is designed in [3]. Sufficient conditions are established to guarantee the exponential convergence of the algorithm.

The aforementioned works require that the communication structures are undirected or at least balanced. Moreover, to eliminate the communication of auxiliary states, the lower bound of local convexity constants are used to establish the convergence of algorithms $[3,6]$. Designing optimization algorithms on a more general communication structure and relaxing the assumptions on the local gradients remain as ongoing research issues.

In this letter, we consider the distributed optimization problem where each agent has a strongly convex cost function with globally Lipschitz gradients. A continuous-time algorithm is presented for unbalanced directed graphs. Sufficient conditions for the convergence are derived based on invariance and Lyapunov stability theory. By introducing a semi-positive definite term to the Lyapunov function and exploring the invariant projection of Laplacian matrix, the requirement of the lower bound of local convexity constants is removed. Finally, we build a experiment on a distributed microcomputer platform to validate the results.

Methodology. Consider a group of $N$ agents. The communication topology among agents is described by the directed graph $\mathcal{G}$. The set of agents is defined as $\mathcal{V}=\{1, \cdots, N\}$. The adjacency ma-

\footnotetext{
*Corresponding author (email: zhengtao.ding@manchester.ac.uk)
} 
trix is defined as $\mathcal{A}=\left[\alpha_{i j}\right] \in \mathbb{R}^{N \times N}$, where $\alpha_{i i}=0$ and $\alpha_{i j}=1$ if the $i$ th agent can get the information from the $j$ th agent, otherwise $\alpha_{i j}=0$. The Laplacian matrix $\mathcal{L}=\left[l_{i j}\right] \in \mathbb{R}^{N \times N}$ associated with $\mathcal{G}$ is defined as $l_{i i}=\sum_{j=1}^{N} a_{i j}$ and $l_{i j}=-a_{i j}$, for $i \neq j$. A directed graph is strongly connected if there exists a directed path from every agent to every other agent.

Throughout this letter, we make following assumptions.

Assumption 1. Each local objective function $f_{i}$ is strongly convex and differentiable with globally Lipschitz gradient, i.e., there exists $K_{i} \in \mathbb{R}_{\geqslant 0}$ such that $\left\|\nabla f_{i}(x)-\nabla f_{i}(y)\right\| \leqslant K_{i}\|x-y\|, \forall x, y \in \mathbb{R}^{n}$.

With Assumption 1, the global objective function $f$ is also strongly convex, and the solution of problem (1) is unique.

Assumption 2. The communication topology $\mathcal{G}$ is strongly connected.

With Assumption 2 and [7, Lemma 2.1], following similar steps in [1], we transform the problem (1) into a minimization problem under a consensus condition.

$$
\begin{aligned}
& \min _{x \in \mathbb{R}^{N n}} \tilde{f}(x)=\sum_{i=1}^{N} f_{i}\left(x_{i}\right), \\
& \text { s.t. }\left(\mathcal{L} \otimes I_{n}\right) x=\mathbf{0}_{N n},
\end{aligned}
$$

where $x_{i} \in \mathbb{R}^{n}$ is the state of $i$ th agent, $x$ is the aggregated variable of $x_{i}$, and $\mathbf{0}_{N n}$ denotes a column vector of size $N n$ with all entries equal to zero. In the following, we will introduce the main results of this letter.

A continuous-time optimization algorithm is designed as

$$
\begin{aligned}
& \dot{x}=-\gamma \nabla \tilde{f}(x)-\alpha\left(\Xi \mathcal{L} \otimes I_{n}\right) x-v, \\
& \dot{v}=\alpha \beta\left(\Xi \mathcal{L} \otimes I_{n}\right) x,
\end{aligned}
$$

where $v_{i} \in \mathbb{R}^{n}$ is the auxiliary state of $i$ th agent, $v$ is the aggregated variable of $v_{i}$, $\gamma, \alpha, \beta \in \mathbb{R}_{>0}$ are constant gains, $\nabla \tilde{f}(x)=$ $\left[\nabla f_{1}\left(x_{1}\right)^{\mathrm{T}}, \nabla f_{2}\left(x_{2}\right)^{\mathrm{T}}, \cdots, \nabla f_{N}\left(x_{N}\right)^{\mathrm{T}}\right]^{\mathrm{T}}$ is the gradient of $\tilde{f}$, and $\Xi$ is defined in [7, Lemma 2.2]

Lemma 1. Under Assumptions 1 and 2, the equilibrium point of $(3)$ satisfying $(\bar{x}, \bar{v}) \in \mathcal{P}_{0}(0)$ is an optimal solution of problem $(2)$, where $\mathcal{P}_{0}(0)=$ $\left\{(x, v) \in \mathbb{R}^{N n} \times \mathbb{R}^{N n} \mid\left(\mathbf{1}_{N}^{\mathrm{T}} \otimes I_{n}\right) v=\mathbf{0}_{n}\right\}$.

Proof. Note that the equilibrium point $(\bar{x}, \bar{v})$ of (3) satisfies

$$
\begin{aligned}
& \bar{x}=\mathbf{1}_{N} \otimes a, \forall a \in \mathbb{R}^{n}, \\
& \bar{v}=-\gamma \nabla \tilde{f}(\bar{x}) .
\end{aligned}
$$

Applying $\left(\mathbf{1}_{N}^{\mathrm{T}} \otimes I_{n}\right) \bar{v}=-\gamma \sum_{i=1}^{N} f_{i}(\bar{x})=\mathbf{0}_{n}$, we have that $(\bar{x}, \bar{v})$ is the optimal solution $\left(x^{\star}, v^{\star}\right) . \triangleleft$
Theorem 1. Under Assumptions 1 and 2, algorithm (3) solves the distributed optimization problem (2) for $(x(0), v(0)) \in \mathcal{P}_{0}(0)$, if $\alpha, \beta, \gamma \in \mathbb{R}_{>0}$ satisfy

$$
\begin{aligned}
& (\delta+1) \gamma \beta-2 \gamma^{2} \bar{K}>0 \\
& (2 \delta+1) \lambda_{2}(\overline{\mathcal{L}}) \alpha \beta-\frac{17}{2}(\delta+1)^{2} \beta^{2}>0
\end{aligned}
$$

where $\delta \in \mathbb{R}_{>0}, \bar{K}=\max \left\{K_{1}, K_{2}, \cdots, K_{N}\right\}$, $\overline{\mathcal{L}}=\Xi \mathcal{L}+\mathcal{L}^{\mathrm{T}} \Xi$ and $\lambda_{2}(\overline{\mathcal{L}})$ denotes the smallest nonzero eigenvalues of $\overline{\mathcal{L}}$.

Proof. Define $\rho=x-\bar{x}, \varrho=v-\bar{v}$. We can get the network dynamics

$$
\begin{aligned}
& \dot{\rho}=-\gamma h-\alpha\left(\Xi \mathcal{L} \otimes I_{n}\right) \rho-\varrho, \\
& \grave{\varrho}=\alpha \beta\left(\Xi \mathcal{L} \otimes I_{n}\right) \rho \text {, }
\end{aligned}
$$

where $h=\nabla \tilde{f}(x)-\nabla \tilde{f}(\bar{x})$.

Consider the following Lyapunov function candidate

$$
\begin{aligned}
V_{2}= & \frac{1}{2} \rho^{\mathrm{T}}\left(\left((\delta+1) \beta \Pi+\delta \beta I_{N}\right) \otimes I_{n}\right) \rho \\
& +\frac{1}{2 \beta}(\beta \rho+\varrho)^{\mathrm{T}}(\beta \rho+\varrho), \\
\text { where } \Pi= & I_{N}-\frac{1}{N} \mathbf{1}_{N} \mathbf{1}_{N}^{\mathrm{T}} .
\end{aligned}
$$

The time derivative of $V_{2}$ along (3) is given by

$$
\begin{aligned}
\dot{V}_{2}= & -(\delta+1) \gamma \beta \rho^{\mathrm{T}} h-(\delta+1) \gamma \beta \rho^{\mathrm{T}}\left(\Pi \otimes I_{n}\right) h \\
& -\frac{(2 \delta+1)}{2} \alpha \beta \rho^{\mathrm{T}}\left(\overline{\mathcal{L}} \otimes I_{n}\right) \rho-(\delta+1) \beta \rho^{\mathrm{T}} \varrho \\
& -(\delta+1) \beta \rho^{\mathrm{T}}\left(\Pi \otimes I_{n}\right) \varrho-\gamma \varrho^{\mathrm{T}} h-\varrho^{\mathrm{T}} \varrho . \quad(5)
\end{aligned}
$$

Note that $\mathcal{P}_{0}(0)$ is positive invariant under (3). Furthermore, for $(x, v) \in \mathcal{P}_{0}(0)$, we have $\rho^{\mathrm{T}} \varrho=$ $\rho^{\mathrm{T}}\left(\Pi \otimes I_{n}\right) \varrho$. Using this fact, we can rewrite $(5)$ as

$$
\begin{aligned}
\dot{V}_{2}= & -(\delta+1) \gamma \beta \rho^{\mathrm{T}} h-(\delta+1) \gamma \beta \rho^{\mathrm{T}}\left(\Pi \otimes I_{n}\right) h \\
& -2(\delta+1) \beta \rho^{\mathrm{T}}\left(\Pi \otimes I_{n}\right) \varrho-\gamma \varrho^{\mathrm{T}} h-\varrho^{\mathrm{T}} \varrho \\
& -\frac{(2 \delta+1)}{2} \alpha \beta \rho^{\mathrm{T}}\left(\overline{\mathcal{L}} \otimes I_{n}\right) \rho \\
\leqslant & -\left((\delta+1) \gamma \beta-2 \gamma^{2} \bar{K}\right) \rho^{\mathrm{T}} h-\frac{1}{2} \varrho^{\mathrm{T}} \varrho \\
& -\left\|2(\delta+1) \beta\left(\Pi \otimes I_{n}\right) \rho+\frac{1}{2} \varrho\right\|^{2}-\left\|\gamma h+\frac{1}{2} \varrho\right\|^{2} \\
& -\left\|\frac{1}{2}(\delta+1) \beta\left(\Pi \otimes I_{n}\right) \rho+\gamma h\right\|^{2} \\
& -\rho^{\mathrm{T}}\left(\left(\frac{(2 \delta+1)}{2} \alpha \beta \overline{\mathcal{L}}-\frac{17}{4}(\delta+1)^{2} \beta^{2} \Pi\right) \otimes I_{n}\right) \rho .
\end{aligned}
$$

The last equality follows the facts that $\Pi \Pi=\Pi$ and $h^{\mathrm{T}} h \leqslant \bar{K} \rho^{\mathrm{T}} h$. 
Since $\overline{\mathcal{L}} \Pi=\Pi \overline{\mathcal{L}}$, by $[8$, Theorem 4.1.6], there exists an orthogonal matrix $U \in \mathbb{R}^{N \times N}$ such that

$$
\begin{aligned}
& \frac{(2 \delta+1)}{2} \alpha \beta \overline{\mathcal{L}}-\frac{17}{4}(\delta+1)^{2} \beta^{2} \Pi \\
= & U\left[\frac{(2 \delta+1)}{2} \alpha \beta\left[\begin{array}{cccc}
0 & 0 & \cdots & 0 \\
0 & \lambda_{2}(\overline{\mathcal{L}}) & \cdots & 0 \\
\vdots & \vdots & \ddots & \vdots \\
0 & 0 & \cdots & \lambda_{N}(\overline{\mathcal{L}})
\end{array}\right]\right. \\
- & \left.\frac{17}{4}(\delta+1)^{2} \beta^{2}\left[\begin{array}{cccc}
0 & 0 & \cdots & 0 \\
0 & 1 & \cdots & 0 \\
\vdots & \vdots & \ddots & \vdots \\
0 & 0 & \cdots & 1
\end{array}\right]\right) U^{\mathrm{T}} .
\end{aligned}
$$

Applying $\rho^{\mathrm{T}} h \geqslant 0,(4)$ and (6), we have $\dot{V}_{2} \leqslant$ 0 . Therefore, we can conclude that the variables $\rho$ and $\varrho$ are bounded. From LaSalle's invariance principle, we have $\lim _{t \rightarrow \infty} \rho^{\mathrm{T}} h=0$ and $\lim _{t \rightarrow \infty} \varrho=\mathbf{0}_{N n}$, which implies that $x_{i}$ converges to the optimal solution of problem (2) as $t \rightarrow \infty$, for $i=1, \cdots, N$.

There always exist $\alpha, \beta, \gamma \in \mathbb{R}_{>0}$ satisfying (4); e.g., by choosing $\beta, \gamma, \delta \in \mathbb{R}_{>0}$ satisfying (4a), we can find sufficiently large $\alpha$ to have (4b). The tunning of $\alpha$ and $\gamma$ is decoupled.

Experiment Validation. A microcomputer platform is used to validate the design of algorithm (3). The microcomputer platform shown in Figure 1 (left) consists of a router and 5 microcomputers. Each microcomputer has a onboard processor Cortex-A53 running at $1.2 \mathrm{GHz}$ and a micro$\mathrm{SD}$ card. The router is used to set up the wireless communication among microcomputers, each of which can get the state information of its neighbors through a $802.11 \mathrm{n}$ wireless LAN that is provided by the onboard chip BCM43438.

Consider a network of 5 agents with local cost functions given by

$$
\begin{array}{ll}
f_{1}=x^{\frac{4}{3}}, & f_{2}=e^{0.2 x}, \\
f_{3}=(x+2)^{2}, & f_{4}=0.1 x^{2}+\frac{x^{2}}{\sqrt{x^{2}+1}}, \\
f_{5}=x^{2}+\ln \left(x^{2}+1\right), &
\end{array}
$$

where $x \in \mathbb{R}$. The initial states $x_{i}(0), i=$ $1,2, \cdots, 5$ are randomly selected within $[0.11,1]$. Then we can calculate the optimal solution $x^{\star}=$ 0.6575 . To satisfy $(x(0), v(0)) \in \mathcal{P}_{0}(0), v_{i}(0)$ are set as $0, \forall i=1,2, \cdots, 5$. The parameters are chosen as $\alpha=6, \beta=1$, and $\gamma=1$. The communication structure $\mathcal{G}$ is shown in Figure 1 (left).

To implement the algorithms (3) on hardware platform, the gradients $\nabla f_{i}$, which include terms $x^{\frac{1}{3}}, \sqrt{x^{2}+1}$ and $e^{0.2 x}$, are approximated by a
Newton iterative method and Taylor series. The accuracy of approximation is set as $10^{-5}$. The integrations are calculated using a forward Euler method. Furthermore the frequency of algorithm (3) is $100 \mathrm{~Hz}$.

The result in Figure 1(right) shows that all the trajectories of states converge to the optimal solution, implying (3) can be implemented on embedded systems with limited computation capability.

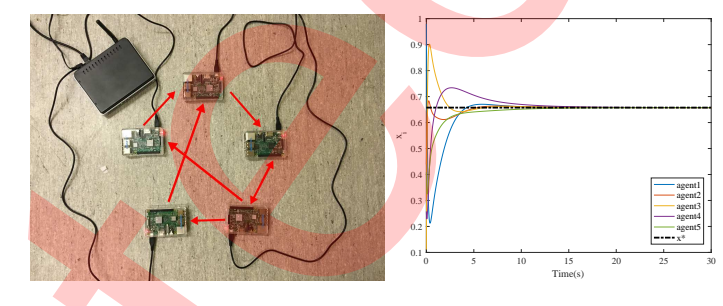

Figure 1 Communication structure $\mathcal{G}$ (left); the agent states $x_{i}, 1,2, \cdots, 5$ (right).

Conclusion. In this letter, we consider the DOP on unbalanced directed graphs. Sufficient conditions for the convergence are established without the knowledge of the lower bound of local convexity constants. The experiment results show that our algorithm can be implemented on embedded systems with limited computation capability.

Acknowledgements This work was supported by the Science and Technology Facilities Council under Grant ST/N006852/1.

\section{References}

1 Li Z, Ding Z, Sun J, Li Z. Distributed adaptive convex optimization on directed graphs via continuous-time algorithms. IEEE Trans Autom Control, 2018, 63: 14341441

2 Gharesifard B, Cortes J. Distributed continuoustime convex optimization on weight-balanced digraphs. IEEE Trans Autom Control, 2014, 59: 781-786

3 Kia S S, Cortes J, Martinez S. Distributed convex optimization via continuous-time coordination algorithms with discrete-time communication. Automatica, 2015, 55: 254-264

4 Wang J, Elia N. A control perspective for centralized and distributed convex optimization. In Proc 50th IEEE Conf Decision Control Eur Control Conf, 2011. 3800-3805

$5 \mathrm{Lu} \mathrm{J}$, Tang C Y. Zero-gradient-sum algorithms for distributed convex optimization: The continuous-time case. IEEE Trans Autom Control, 2012, 57: 2348-2354

6 Yang S, Liu Q, Wang J. Distributed optimization based on a multiagent system in the presence of communication delays. IEEE Trans Syst, Man, Cybern, Syst, 2017, 47: 717-728

7 Mei J, Ren W, Chen J. Distributed consensus of secondorder multi-agent systems with heterogeneous unknown inertias and control gains under a directed graph. IEEE Trans Autom Control, 2016, 61: 2019-2034

8 Horn R A, Johnson C R. Matrix analysis. Cambridge university press, 1985 . 172-173 